\title{
Transnationale Rationalitäten, nationale Traditionen: ein Dilemma der Regierung im Mehrebenensystem
}

von Richard Münch

Die fortschreitende europäische Integration und die entsprechende Entwicklung eines Mehrebenensystems forcieren zwei miteinander verwobene Trends: (1) die Verwissenschaftlichung und (2) die Vermarktlichung des Regierungs- und Verwaltungshandelns. Das bringt transnationale Rationalitäten und nationale (wie auch regionale und lokale) Traditionen miteinander in Konflikt. Die Vielfalt von Traditionen wird in der Tendenz dem Einheitsmaß transnationaler Rationalitätsmodelle geopfert. Das wird am Beispiel der Vermarktlichung von Professionen und der damit einhergehenden Erosion der treuhänderischen Verantwortung für das Gemeinwohl deutlich gemacht. Am Ende stellt sich die Frage, durch welche institutionellen Vorkehrungen diesem Konflikt Rechnung getragen werden kann.

The progressing European integration and the corresponding development of a multilevel-system promote two interconnected trends (1) the scientification and (2) the marketisation of governement and administration. This development involves a conflict between transnational rationalities and national (as well as regional and local) traditions. The diversity of traditions tends to be sacrificed to the uniform measure of transnational rationality models. This conflict is demonstrated using the marketisation of professions as an example, which is accompanied by the erosion of the fiduciary responsibility for the common good. In conclusion, the question is raised as to what institutional devices might be appropriate to account for this conflict.

Die Transnationalisierung, in besonders forcierender Gestalt die Europäisierung nationaler Gesellschaften, entmachtet lokale Autoritäten und delegitimiert lokale Praktiken und ersetzt sie durch die Herrschaft von Wissenseliten und durch die globale Geltung ,wissenschaftlich“ begründeter Rationalitätsmodelle. Und sie entlässt die Individuen aus lokalen und nationalen Bindungen sowie aus hierarchischer Kontrolle, um sie der Selbstbehauptung im Wettbewerb auf alle Lebensbereiche durchdringenden Märkten zu überlassen. Jenseits territorial gebundener Herrschaft stößt die hierarchische Organisation des gesellschaftlichen Lebens an enge Grenzen. Das bereitet der Koordination des menschlichen Han- 
delns durch Märkte einen fruchtbaren Boden. Das Leben spielt sich immer mehr in einer Hayek'schen Welt der offenen Koordination durch Märkte ab. ${ }^{1}$ Für die in der Gegenwart intensivierten Trends der Verwissenschaftlichung und Vermarktlichung des Regierungs- und Verwaltungshandelns und der Individualisierung der Lebensführung und Verteilung von Lebenschancen gibt es demnach jenseits zufälliger politischer Konstellationen eine tiefere Ursache: die Transnationalisierung der Regierung und Verwaltung und der Vergesellschaftung. Es sind demnach zwei Prozesse genauer zu betrachten: (1) die Verwissenschaftlichung und (2) die Vermarktlichung des Regierungs-. und Verwaltungshandelns als Resultanten der Transnationalisierung des gesellschaftlichen Geschehens und damit einhergehend der Entwicklung eines Mehrebensystems.

\section{Die Verwissenschaftlichung von Regierung und Verwaltung}

Wissenschaftliches Wissen setzt sich gegen den Widerstand historisch gewachsener Lebenswelten und ihre traditionale Legitimität durch. Es unterwirft die Lebenswelten einem fortlaufenden Prozess der Delegitimation ihrer Traditionen. ${ }^{2}$ Historisch gewachsene Muster von Wissen, Normen und Verhaltensweisen werden mit dem globalen Siegeszug der westlichen Wissenschaft zunehmend hinterfragt und verlieren ihre Selbstverständlichkeit. Sie gelten nicht mehr für sich selbst in ihrer eigenen Qualität, sondern müssen ihre instrumentelle Rationalität zur Erreichung von Zwecken beweisen, die sich im Kontext der Weltkultur als allgemein verbindlich durchgesetzt haben: der Fortschritt der Erkenntnis und die Steigerung des Wohlstands als Konkretisierung des instrumentellen Rationalismus. Dazu gehört auch die Bewältigung ökologischer Probleme soweit sie eine Gefahr für die Steigerung des Wohlstands sind.

Mit John Meyers Ansatz des Neoinstitutionalismus lässt sich die globale Ausbreitung wissenschaftlich begründeter Modelle rationaler Praxis durch das Entstehen einer westlich geprägten Weltkultur erklären, nach deren Prinzipien Akteure (Individuen, Organisationen, Nationalstaaten) handeln müssen, um einen legitimen Status als rational handelnde Akteure zu erlangen. Von besonderer Bedeutung dafür ist die Herausbildung von globalen Expertennetzwerken und Internationalen Organisationen als verbindliche Definitionsmacht ausübende

1 Hayek, F.A.v.: Die Ergebnisse menschlichen Handelns, aber nicht menschlichen Entwurfs, in: ders.: Freiburger Studien. Gesammelte Aufsätze, Tübingen, 1969, 97-107.

2 Drori, G. u. a.: World Society and the Authority and Empowerment of Science, in: dies. (Hg.): Science in the Modern World Polity, Stanford, 2003, 23-42. 
Instanzen. ${ }^{3}$ In Anlehnung an die Begrifflichkeit von George Herbert Mead können sie als kulturelle Andere verstanden werden, die ohne eigene Interessen allgemeine Prinzipien zur Geltung bringen, die zu verbindlichen Leitlinien für Individuen, Organisationen und Nationalstaaten werden. ${ }^{4}$ Die europäische Integration kann als ein besonders verdichteter Prozess verstanden werden, in dem die Leitlinien der internationalen Zusammenarbeit und der wirtschaftlichen Integration verbindlichen Status erlangt haben, von der Europäischen Kommmission repräsentiert, in den Ausschüssen der Komitologie kleingearbeitet und vom Europäischen Gerichtshof (EuGH) durchgesetzt werden. ${ }^{5}$

Bezogen auf demokratisches Regieren bedeutet die Verwissenschaftlichung von Regierung und Verwaltung durch ihre Transnationalisierung die Entmachtung von Parlamenten, Parteien und Verbänden und einen Machtzuwachs von wissenschaftlichen Experten, Beratern und Kommissionen. Die höchste Legitimationsstufe dieser Machtverschiebung wird im wissenschaftlichen Diskurs über die europäische Form der governance erreicht. Im wissenschaftlichen Diskurs wird die Machtverschiebung in der zunehmenden Bedeutung von Theorien reflektiert, die insbesondere in der Europäischen Union die Konturen eines regulativen Staates erkennen, der den distributiven nationalen Wohlfahrtsstaat ablöst. Die Legitimität der Entscheidungen des regulativen Staates gründet nicht mehr in der demokratischen Willensbildung, sondern in der Treuhänderschaft der Experten ${ }^{6}$, der Deliberation ${ }^{7}$ bzw. der Ablösung von bargaining durch arguing ${ }^{8}$ in den Ausschüssen der europäischen Komitologie. Diese wissenschaftliche Literatur liefert die Legitimationsgrundlagen für die Entmachtung der Parlamente, Parteien und Verbände durch die Experten. In allen Fragen der Gesellschaftspolitik üben heu-

3 Meyer, J.W.: Weltkultur. Wie die westlichen Prinzipien die Welt durchdringen, hg.v. Krücken, G., Frankfurt/M., 2005.

4 Mead, G.H.: Geist, Identität und Gesellschaft aus der Sicht des Sozialbehaviorismus, Frankfurt/M., 1968.

5 Münch, R.: Constructing a European Society by Jurisdiction, in: European Law Journal, 14/5 (2008), 519-541; ders.: Die Konstruktion der europäischen Gesellschaft Zur Dialektik von transnationaler Integration und nationaler Desintegration, Frankfurt/New York, 2008.

6 Majone, G.: Regulating Europe, London, 1996.

7 Joerges, C./Neyer, J.: From Intergovernmental Bargaining to Deliberative Political Processes: The Constitutionalization of Comitology, in: European Law Journal, 3/3 (1997), 273-299.

8 Gehring, T.: Bargaining, Arguing and Functional Differentiation of Decision-Making: The Role of Committees in European Environmental Process Regulation, in: Joerges, C./Vos, E. (Hg.): European Committees: Social Regulation, Law and Politics, Oxford, 1999, 195-217. 
te insbesondere Ökonomen entscheidende Definitionsmacht aus. ${ }^{9}$ Sie sind die Experten für eine wissenschaftlich begründete Sozialpolitik und gelangen im transnationalen Kontext in eine dominante Position, während sie sich im nationalen Feld der Sozialpolitik lange Zeit in einer nachrangigen Stellung gegenüber den Partei- und Verbandsvertretern befanden. Die von der Lissabon-Strategie der EU forcierte Entwicklung der Wissensgesellschaft verstärkt diese Tendenz und ersetzt zunehmend die demokratische Form der Regierung und Verwaltung durch eine wissenschaftlich angeleitete. August Comtes positives Zeitalter ${ }^{10}$ wird damit in der Wissensgesellschaft tatsächlich zur Wirklichkeit. Die Entmachtung von Parlamenten, Parteien und Verbänden durch die Verwissenschaftlichung von Regierung und Verwaltung vergrößert indessen unintendiert den Spielraum der von den Massenmedien und ihrer Gesetzmäßigkeiten der Aufmerksamkeitsökonomie gestalteten politischen Kommunikation. ${ }^{11}$ Politisches Entscheiden verliert dadurch erst recht an Strukturierung durch feststehende, in Parlamenten, Parteien und Verbänden eingewurzelte Konfliktstrukturen und wird dadurch umso mehr dem freien Zusammenspiel von wissenschaftlicher Expertise und medialer Aufmerksamkeitserzeugung unterworfen. An die Stelle der Aushandlung von Kompromissen zwischen den Vertretern der betroffenen Interessengruppen tritt das Regieren mit Zahlen ${ }^{12}$, Tabellen und Kuchendiagrammen sowie die Messung des Regierungserfolgs an diesen Zahlen in der massenmedialen Kommunikation. Wissenschaftliche Experten gewinnen die Macht, mit mehr oder weniger abgesicherten Gutachten zu Ökologie, Bildung, Renten, sozialer Sicherheit und wirtschaftlichem Wachstum die mediale Aufmerksamkeit für Reformen zu erzeugen, die innerhalb der traditionalen Konfliktstrukturen von Parlamenten, Parteien und Verbänden scheitern würden. Neu ist, dass sich die Experten nicht mehr allein auf die Kommissionsarbeit beschränken, sondern direkt die Öffentlichkeit mobilisieren, um ihr wissenschaftliches Kapital symbolisch aufzuwerten und ihre Verhandlungsposition in der Kommissionsarbeit zu untermauern.

Gleichwohl bedeutet die Verwissenschaftlichung von Regierung und Verwaltung nicht zwangsläufig eine Steigerung der funktionalen Effektivität der entspre-

9 Fourcade, M.: The Construction of a Global Profession: The Transnationalization of Economics, in: American Journal of Sociology, 112/1 (2006), 145-194.

10 Comte, A.: Cours de philosophie positive (1830-42), hg.v. Serres, M./Gagognet, F./Sinacour, A., Paris, 1975.

11 Münch, R.: Dialektik der Kommunikationsgesellschaft, Frankfurt/M., 1991; Franck, G.: Ökonomie der Aufmerksamkeit: ein Entwurf, München, 1998.

12 Porter, T. M.: Trust in Numbers. The Pursuit of Objectivity in Science and Public Life, Princeton, 1996. 
chenden Reformen. Isomorphe Prozesse der Unterwerfung unter normativen Druck zur Erlangung von Anerkennung und legitimem Status innerhalb der Wissenschaft und in der Umsetzung ihrer Ergebnisse in politische Entscheidungen haben zur Folge, dass in erheblichem Maße global diffundierte Reformschablonen ohne Kenntnis ihrer realen Wirkungen in lokalen Kontexten Anwendung finden und einerseits die Entkopplung der praktischen Aktivitätsstruktur von der rationalisierten Formalstruktur ${ }^{13}$, andererseits nichtintendierte Effekte den mit einer Reform verbundenen Zielen entgegenwirken. Eine Konsequenz der verwissenschaftlichten Regierung und Verwaltung ist deshalb die Verstärkung der Paradoxie des instrumentellen Rationalismus, die sich in der zunehmenden und beschleunigten Erzeugung nichtintendierter Konsequenzen von Reformen äußert, die erneut zu einem Wachstum und einer Beschleunigung von Reformen zwingen. Gemessen an herkömmlichen Kriterien der Demokratie entsteht auf diese Weise eine Form der nichtlegitimen Herrschaft.

Bezogen auf die Organisation des menschlichen Handelns in der öffentlichen Verwaltung und in der privaten Unternehmung findet eine Entbürokratisierung von Herrschaft statt. An die Stelle der Herrschaft des Rechts und der Ausübung von Entscheidungskompetenzen durch Anwendung des Rechts bzw. durch Ausführung gegebener Aufgaben treten New Public Management in der Verwaltung und profit center in der Unternehmung, jeweils in Verbindung mit dem Instrument der Zielvereinbarung und zweckbestimmten Erfolgskontrolle. Der gewachsene Handlungsspielraum der Organisationsmitarbeiter unterliegt dabei einer umso totaleren Erfolgskontrolle anhand der Erfüllung vorher definierter Kennziffern. Es setzt sich eine neue, rationalisierte Gouvernementalität durch, die von der Dialektik wachsender Entscheidungsspielräume und gleichzeitig wachsender, zur Totalität neigenden Kontrolle geprägt wird. Der größere Freiheitsgrad muss mit der Unterwerfung unter umfassendere Kontrolle bezahlt werden.

An dieser Stelle gibt es eine bemerkenswerte Nähe des Meyer'schen WeltkulturAnsatzes zu Foucaults Studien zur Geschichte der Gouvernementalität ${ }^{14}$. Nach Foucault kann man das europäische Binnenmarktprogramm als eine aktuelle Entwicklungsstufe der Gouvernementalisierung des Staates begreifen. Demnach wird die souveräne staatliche Herrschaft über ein Territorium (Stufe 1) und die staatliche Disziplinarmacht (Stufe 2) mit dem europäischen Binnenmarktpro-

13 Meyer, J.W./Rowan, B.: Institutionalized organizations: Formal structure as myth and ceremony, in: American Journal of Sociology, 83/2 (1977), 340-363.

14 Foucault, M.: Geschichte der Gouvernementalität, 2 Bde., Frankfurt/M., 2006. 
gramm durch ein entterritorialisiertes Regieren (Stufe 3) abgelöst, dessen Reichweite durch die Symbiose von Markt und Recht, der Nutzung wirtschaftlicher Freiheiten und der Ausübung ziviler Rechte begrenzt wird. Die Kunst des europäischen Regierens liegt demnach darin, den Punkt zu treffen, an dem ein Maximum der wirtschaftlichen und zivilen Freiheiten so koordiniert wird, dass sie alle zusammen bestehen können, ohne sich gegenseitig aufzuheben. Die Instanz, die maßgeblich diese Justierung der Freiheiten betreibt, ist der Europäische Gerichtshof. Die europäische Rechtssetzung hat im Vertragswerk der EU eine klar definierte Leitlinie der Gewährleistung des freien Verkehrs von Waren, Dienstleistungen, Kapital und Personen und der Beseitigung jeglicher Art der Diskriminierung im Marktzutritt als Grundlage der autonomen Lebensführung des Individuums. Darüber hinaus sorgt die zunehmend zum Einsatz kommende Offene Methode der Koordinierung (OMK) ohne öffentliche Debatte und demokratische Willensbildung für eine quasi natürliche Selektion von ,best practices “ im institutionellen Wettbewerb.

Die Transnationalisierung des gesellschaftlichen Geschehens und das Entstehen eines Mehrebenensystems schaffen also erstens auf der semantischen Ebene Legitimationsgrundlagen für die fortschreitende Verwissenschaftlichung von Regierung und Verwaltung mit einem Machtzuwachs wissenschaftlicher Expertise und einem Machtverlust von Parlamenten, Parteien und Verbänden, und damit geht gleichzeitig ein Machtzuwachs der politischen Medienkommunikation und ihrer Gesetzmäßigkeiten der Aufmerksamkeitsökonomie einher. Zweitens erzeugen die Lissabon-Strategie und ihre Umsetzung in Verfahren der OMK in formaler Hinsicht auf der semantischen Ebene Legitimationsgrundlagen für die Durchsetzung von neuen rationalisierten Formen der Gouvernementalität und ihrer Dialektik der Erweiterung von Entscheidungsspielräumen bei gleichzeitiger Totalisierung der Kontrolle über das Individuum. In inhaltlicher Hinsicht wird die Verwissenschaftlichung von Regierung und Verwaltung im Kontext ihrer Transnationalisierung von zwei Erscheinungsformen der Paradoxie des instrumentellen Rationalismus geprägt: (1) Wachstum und Beschleunigung von Reformprozessen durch nichtintendierte Folgen, (2) Totalisierung der Kontrolle über die Mitarbeiter von Organisationen in Verbindung mit der Ausweitung ihrer Entscheidungsspielräume.

\section{Die Vermarktlichung von Regierung und Verwaltung}

Die Beseitigung von Staatsmonopolen, die Privatisierung von Staatsbetrieben, Deregulierung und die Ersetzung von Hierarchien durch Märkte schaffen in 
Europa und darüber hinaus in aller Welt durch alle Staaten hindurch eine Ordnung, die nicht auf der Disziplinierung durch den Staat beruht, sondern auf der disziplinierenden Wirkung des Wettbewerbs. Maximaler Handlungsspielraum geht dabei mit maximaler Selbstdisziplinierung durch Techniken des Selbst einher. Wer dabei Hilfe braucht, findet reichhaltigen Expertenrat, von der Ratgeberliteratur für den Alltagsgebrauch des einzelnen Individuums bis zur Beratungsindustrie für Unternehmen, Kirchen, Verbände, Schulen und Staaten und zu den Expertenstäben internationaler Regierungs- und Nichtregierungsorganisationen. An dieser Stelle ergänzen Meyers „kulturelle Andere“, die uneigennützigen, wissenschaftlich begründeten Expertenrat geben, Foucaults Kunst des Regierens als umsichtiges Ausbalancieren eines sich weitgehend selbst regulierenden Prozesses. $^{15}$

Der universale Individualismus setzt sich gegen den Partikularismus und gegen den Kollektivzwang traditionaler Lebenswelten durch. Die partikularen Solidaritäten historischer Gemeinschaften verlieren im Kontext der Weltkultur ihre Legitimationsgrundlage. Die Bevorzugung lokaler, regionaler oder nationaler Gemeinschaften ist nicht mehr $\mathrm{zu}$ rechtfertigen. Warum die Mitglieder der hochentwickelten Industrieländer kollektiv einen höheren Lebensstandard teilen sollen als die Mitglieder von Entwicklungsländern, lässt sich nicht mit zwingenden Gründen beantworten. Das Recht auf Teilhabe am erwirtschafteten Wohlstand gilt in der Weltgesellschaft endgültig universell für alle Menschen. Die Menschen- und Bürgerrechte können niemandem auf der Erde vorenthalten werden. Die Staatsbürger der Nationalstaaten sehen sich immer mehr gezwungen, ihre Rechte auch mit Nichtbürgern (Zuwanderern) zu teilen. ${ }^{16}$ Im europäischen Kontext gilt das zunächst in einem durch Rechtssetzung und Rechtsprechung immer umfassender verwirklichten Sinn für alle Unionsbürger. Mit der Entsolidarisierung lokaler, regionaler und nationaler Gemeinschaften und der Solidarisierung transnationaler Beziehungen im Kontext der transnationalen Universalisierung von Rechten geht die zunehmende Individualisierung der Lebensführung und der Lebenschancen einher. Die Europäisierung des Sozialraumes von Wirtschaft, Recht und Verfassung befreit das Individuum einerseits aus partikularen, nationalen Bindungen und Kollektivzwängen, setzt es aber

15 Vgl. Lemke, T.: Eine Kritik der politischen Vernunft. Foucaults Analysen der modernen Gouvernementalität, Hamburg, 1997; ders.: Neoliberalismus, Staat und Selbsttechnologien. Ein kritischer Überblick über die governmentality studies, in: Politische Vierteljahrschrift, 41/1 (2000), 31-47; Bröckling, U.: Das unternehmerische Selbst, Frankfurt/M., 2007.

16 Soysal, Y.: Limits of Citizenship: Migrants and Postnational Membership in Europe, Chicago, 1994. 
gleichzeitig einem offeneren Wettbewerb aus, wo es vorher durch nationale Vorkehrungen geschützt war. Die Befreiung des Menschen aus nationalen Kollektivzwängen unterwirft ihn deshalb umso mehr den äußeren Zwängen des Marktes. Das ist die Paradoxie des universalen Individualismus. Gemessen an den Institutionen des nationalen Wohlfahrtsstaates, ergibt sich dementsprechend im Kontext der Transnationalisierung des gesellschaftlichen Geschehens eine Rekommodifizierung der Lebensführung und Lebenschancen des Individuums.

Die entsprechende Stärkung der Marktkräfte gegen traditionale Solidaritäten bringt auch eine Restrukturierung von sozialer Ungleichheit in der Verteilung von Lebenschancen auf Nationen, Regionen und Bevölkerungsschichten mit sich. Die Ungleichheit der Lebenschancen, gemessen am Bruttoinlandsprodukt pro Kopf, zwischen den Nationen nimmt tendenziell ab, während sie innerhalb der Nationen tendenziell wächst, soweit die nationalen Beharrungskräfte an Wirkungsmacht verlieren. ${ }^{17}$ In regionaler Hinsicht findet eine Bewegung der Zentralisierung und Peripheralisierung statt. Der Wohlstand konzentriert sich zunehmend auf die aktiven Wirtschaftsregionen, während die nicht in deren Aktivitäten einbezogenen Regionen in die Peripherie verdrängt werden und verarmen. ${ }^{18}$ Sie können weniger als zuvor auf Verfahren des nationalen Finanzausgleichs zwischen Regionen rechnen. Die Sozialstruktur erfährt eine neue Differenzierung. Sie ist durch Elitenbildung an der Spitze, die Normalisierung von Lebensführung und Lebenschancen in der Mitte und die Marginalisierung einer Unterschicht gekennzeichnet. Bildungs-, Beschäftigungs- und Sozialpolitik differenzieren sich dementsprechend zunehmend aus, indem sie eigene Programme für Bildung, Beschäftigung und soziale Sicherheit der Eliten (risikoreicheres Leben mit Spitzeneinkommen), der Normalbürger (das disziplinierte Leben in der Mitte der Gesellschaft) und der neuen Unterschicht (Verwaltung von Exklusion) entwickeln. Bildung wird beispielsweise differenziert in Elitestudiengänge, macdonaldisierte BA-Studiengänge für die Masse der Normalbürger und kompensatorische Erziehung für die Kinder der neuen Unterschicht. Die Paradoxie des universalen Individualismus kommt darin zum Ausdruck, dass die Befreiung des Individuums aus partikularen Solidaritäten und Kollektivzwängen mit dessen Unterwerfung unter die Zwänge globaler Interdependenzen, insbesondere des Marktes einhergeht. Ebenso bringt die Angleichung der Chancen wachsende

17 Firebaugh, G.: The New Geography of Global Income Inequality, Cambridge, MA, 2003.

18 Heidenreich, M.: Territoriale Ungleichheiten in der erweiterten EU, in: Kölner Zeitschrift für Soziologie und Sozialpsychologie, 55/1 (2003), 1-28. 
Resultatsungleichheit und eine entsprechend stärkere Ausdifferenzierung einer transnationalen Wissenselite und einer marginalisierten neuen Unterschicht aus einer der Normalisierung und Standardisierung des Lebens unterworfenen, schrumpfenden Mittelschicht mit sich.

Diese weltgesellschaftlich angetriebene, in der EU europäisch konkretisierte kulturelle Entwicklungsdynamik findet allerdings nicht in einem luftleeren Raum statt. Sie wird maßgeblich durch die ökonomische Dynamik der wachsenden internationalen Arbeitsteilung getragen, die von sich aus Formen der nationalstaatlich gestalteten kollektiven (demokratischen und bürokratischen) Regierung und Verwaltung und kollektiv organisierten Lebensführung und Verteilung von Lebenschancen in ihrer funktionalen Effektivität untergräbt und dafür Formen der experten- und markt- bzw. anreizgesteuerten Regierung und Verwaltung und der individualisierten Lebensführung und Verteilung von Lebenschancen strukturell prämiert. Das äußert sich darin, dass auf der transnationalen europäischen Ebene diese Modelle von Regierung und Verwaltung, Lebensführung und Verteilung von Lebenschancen als kulturelles Skript in den Vordergrund treten und in den Mitgliedstaaten mit dem Grad ihrer wirtschaftlichen, rechtlichen, kommunikativen und kulturellen Integration in die EU die Erwartungs- und Legitimationsstrukturen prägen. Das zeigt sich in einer entsprechenden Differenzierung der Mitgliedstaaten nach der Dauer ihrer Mitgliedschaft, nach dem Grad ihrer EUVerflechtung, ihres wirtschaftlichen Entwicklungsniveaus und des Bildungsniveaus der Bevölkerung. ${ }^{19}$ Von der Seite der Mitgliedstaaten ergibt sich zusätzlich eine Differenzierung der Anpassungsfähigkeit nach dem Grad, in dem sie von kollektiven gouvernementalen, korporativen oder familialen Traditionen bestimmt werden, etwa im Sinne der Differenzierung in Kapitalismus- oder Wohlfahrtsregime-Typen. Deshalb muss der semantische Wandel immer im Verhältnis zur Beharrungskraft der kollektivistischen Traditionen von Regierung und Verwaltung, Lebensführung und Verteilung von Lebenschancen gesehen werden.

Um den gegenwärtigen, von der europäischen Wirtschaftsintegration forcierten Schub der Individualisierung von Lebensführung und Lebenschancen verstehen und ursächlich erklären zu können, reicht der Meyer'sche Weltkultur-Ansatz nicht aus. Er kann nicht begreifbar machen, warum sich in der Gegenwart eine Rekommodifizierung der Lebensführung und Lebenschancen vollzieht, und zwar

19 Gerhards, J.: Kulturelle Unterschiede in der Europäischen Union, Wiesbaden, 2005. 
durch alle wohlfahrtsstaatlichen Formen ihrer Dekommodifizierung ${ }^{20}$ hindurch. Um diesen kulturellen Wandel erklären zu können, muss das Augenmerk auf aktuelle, im System der Wohlfahrtsstaaten für eine begrenzte Zeit gezähmte, treibende Kräfte gerichtet werden. Will man ein tieferes Verständnis dieses Vorgangs gewinnen, als es von dem Verweis auf die im Zuge der Marktöffnung schwindende staatliche Handlungskapazität geboten wird, kann die Rückbesinnung auf Durkheims Studie zur Arbeitsteilung hilfreiche Dienste leisten. ${ }^{21}$ Demnach erzwingen die mit dem Ausbau von Transport- und Kommunikationsmitteln schrumpfenden Distanzen grenzüberschreitend zunehmende Spezialisierung, anderenfalls herrscht der Krieg aller gegen alle. Diese Lektion haben die Pioniere der europäischen Integration nach dem Zweiten Weltkrieg gelernt und haben genau jenen Weg eingeschlagen, den Durkheim schon vor gut 120 Jahren in Gestalt einer aus der grenzüberschreitenden Arbeitsteilung hervorgehenden europäischen Gesellschaft vorgezeichnet sah. In diesem Prozess geht die Annäherung der Nationen aneinander mit ihrer zunehmenden inneren Differenzierung (und Stratifikation) sowie mit der Individualisierung von Identitäten, Lebensführung und Lebenschancen einher. In Foucaults Studien zur Geschichte der Gouvernementalität findet sich eine ganz ähnliche Deutung der europäischen Integration als Projekt der Stärkung des Individuums bei gleichzeitigem Zurücktreten des Staates und der Ersetzung seiner territorialen Herrschaft durch einen sich selbst organisierenden Markt, den es politisch lediglich auszubalancieren gilt. ${ }^{22}$ Die Leitlinie eines global geöffneten Europas, das an die Stelle des Nullsummenspiels territorialer Kämpfe die gegenseitige Bereicherung durch Handel setzt, hat in den Augen Foucaults schon Adam Smith gelegt. ${ }^{23}$

\section{Machtverschiebungen, institutioneller und ideeller Wandel}

Die auf der Linie von Meyer konstatierte Transformation von Regieren und gesellschaftlicher Ordnung im Zuge ihrer Europäisierung lässt sich indessen nicht

20 Esping-Andersen, G.: The Three Worlds of Welfare Capitalism, Cambridge, 1990.

21 Durkheim, É.: Über soziale Arbeitsteilung. Studie über die Organisation höherer Gesellschaften (1893), Frankfurt/M., 1992; Münch, R.: Offene Räume. Soziale Integration diesseits und jenseits des Nationalstaats, Frankfurt/M., 2001; ders.: Die Konstruktion der europäischen Gesellschaft, a.a. O.; Münch, R./Büttner, S.: Die europäische Teilung der Arbeit. Was können wir von Emile Durkheim lernen?, in Heidenreich, M. (Hg.): Die Europäisierung sozialer Ungleichheit. Zur transnationalen Klassen- und Sozialstrukturanalyse, Frankfurt/New York, 2006, 65-107.

22 Vgl. Genschel, P./Zangl, B.: Metamorphosen des Staates - vom Herrschaftsmonopolisten zum Herrschaftsmanager, in: Leviathan, 36/3, (2008), 430-454.

23 Foucault, M., a.a.O., 83-91. 
zureichend begreifen, ohne einen Blick auf die damit einhergehenden Machtverschiebungen zu werfen. Das ist der Ort, an dem sich die Einbeziehung von Bourdieus Feld- und Kapitaltheorie ${ }^{24}$ anbietet.

Der konstatierte gesellschaftliche Wandel findet auf drei Ebenen statt: (1) Machtverschiebungen zwischen Akteursgruppen im diskursiven Feld, (2) Veränderung von Institutionen, (3) Bedeutungswandel grundlegender Ideen. Der gesellschaftliche Wandel schreitet in der Interdependenz zwischen diesen drei

Abbildung 1: Die Überlagerung des nationalen Feldes der Politik durch das transnationale Feld

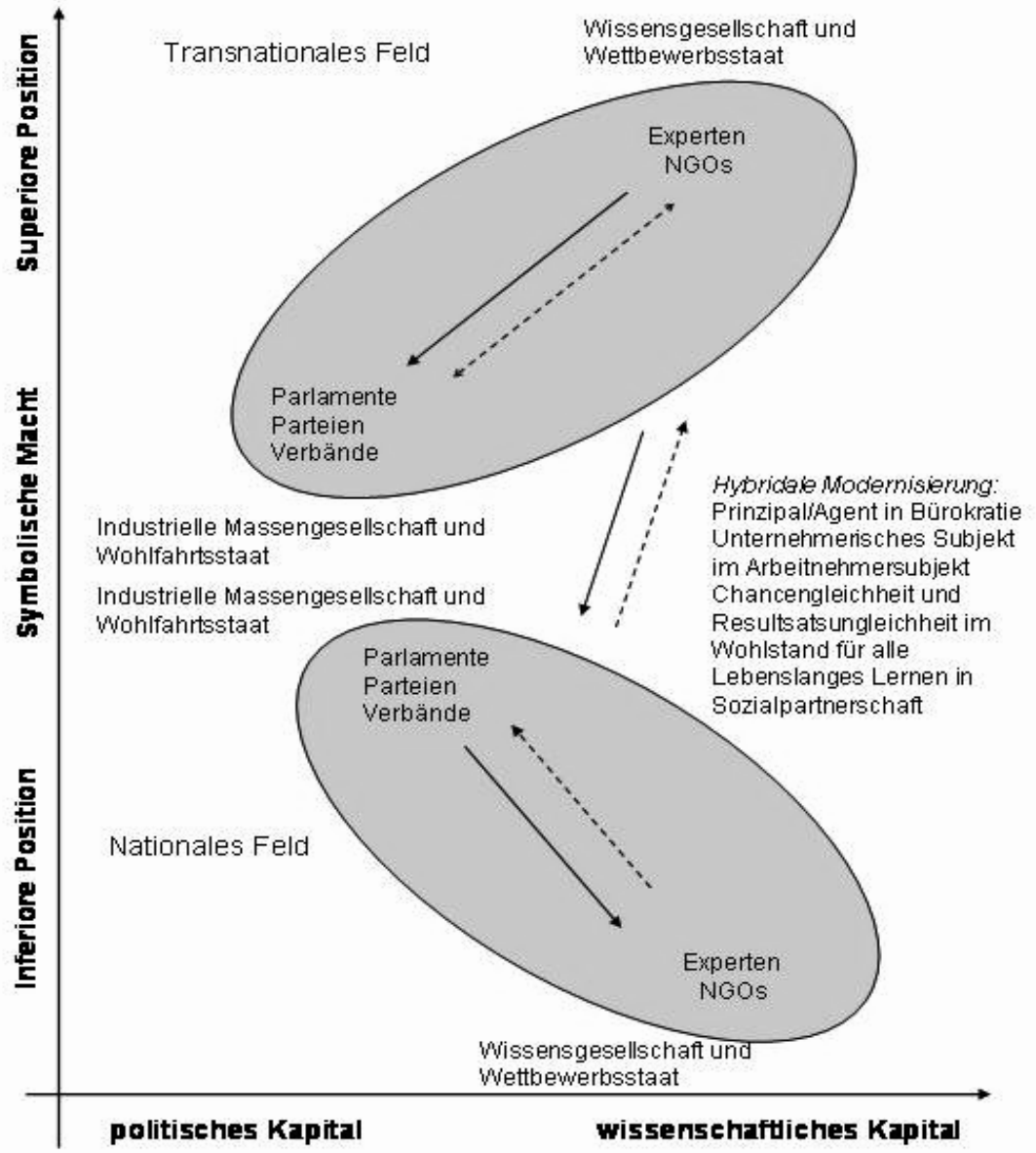

24 Bourdieu, P.: Praktische Vernunft. Zur Theorie des Handelns, Frankfurt/M., 1998; ders.: Der Staatsadel, Konstanz, 2004. 
Ebenen voran. Dabei geht von Machtverschiebungen eine gewisse Dynamik aus, die auf Veränderungen auf der institutionellen und kulturellen (ideellen) Ebene hinwirkt. Je mehr sich auf der kulturellen Ebene neue Bedeutungen von Ideen festgesetzt haben und in einem neuen Paradigma der gesellschaftlichen Selbstbeschreibung fixiert worden sind, umso mehr gewinnen die entsprechenden neuen Institutionen und Machtverhältnisse an Legitimität, Festigkeit und Durchsetzungskraft, während die alten Institutionen und Machtverhältnisse weiter in den Hintergrund gedrängt werden.

Von entscheidender Bedeutung ist die zunehmende Überlagerung des nationalen Feldes der Politik durch das transnationale und die Verschiebung der Gewichte weg vom politischen Kapital (Entscheidungsmacht) und hin zum wissenschaftlichen Kapital (Wissen, Expertise) sowie die Verlagerung der symbolischen Macht (Anerkennung, Definitionsmacht) von den nationalen $\mathrm{zu}$ den transnationalen Eliten. Das Ergebnis dieser Überlagerung sind in aller Regel Formen der hybridalen Modernisierung. Es werden Prinzipal-Agenten-Beziehungen in Bürokratien, Chancengleichheit in Traditionen der Resultatsgleichheit und des Wohlstands für alle und Aspekte des lebenslangen Lernens in die kollektive Daseinsvorsorge eingebaut. Die Symbiose von industrieller Massengesellschaft und Wohlfahrtsstaat wird von einer neuen Symbiose von Wissensgesellschaft und Wettbewerbsstaat abgelöst (vgl. Abbildung 1).

Es ist ein Machtverlust von Parlamenten, Parteien und Verbänden und ein Machtgewinn von wissenschaftlichen Experten und Massenmedien zu beobachten. Die Zahl, die Häufigkeit der Inanspruchnahme und das Gewicht von Experten, Expertengutachten und Expertenkommissionen wachsen; transnational erzeugtes Expertenwissen beeinflusst häufiger und nachhaltiger nationale politische Maßnahmen. ${ }^{25}$ Es häufen sich die Fälle, in denen massives Auftreten international vernetzter Experten die internationale und nationale Politik direkt über die Massenmedien unter Druck setzt (Liberalisierung des Welthandels, Wettbewerbsordnung der EU, Ozon-Regime, Klimaabkommen, PISA, Bologna). In der Sozialpolitik ist diese Machtverschiebung die Grundlage für die zunehmende

25 Martens, K./Wolf, K.D.: Paradoxien der Neuen Staatsräson. Die Internationalisierung der Bildungspolitik in der EU und der OECD, in: Zeitschrift für Internationale Beziehungen, 13/2 (2006), 145-176. 
Dominanz ökonomischer Modelle. Bürokratische Strukturen werden in „Prinzipal-Agenten-Beziehungen“" umgewandelt. Es ergibt sich ein Machtzuwachs auf beiden Seiten derart, dass Agenten mehr Handlungsspielräume und Prinzipalen mehr Möglichkeiten der Erfolgskontrolle gegeben werden. Dadurch wächst die Macht in der Beziehung überhaupt, was einerseits das Handlungspotential steigert, andererseits aber auch das Konfliktpotential und die Häufigkeit und Härte der Konfliktaustragung. Die von Mancur Olson prognostizierte und lange Zeit beklagte institutionelle Sklerose der demokratischen Wohlfahrtsstaaten hat einer erstaunlichen Dynamik des institutionellen Wandels Platz gemacht. Dieser Wandel hat sich überall in die Richtung von Liberalisierung, Deregulierung und Privatisierung bewegt. ${ }^{26}$

\section{Transnationale Rationalität stößt auf nationale Traditionen: Die Ersetzung von professioneller Treuhänderschaft durch Marktwettbewerb}

Ein Feld, auf dem die transnationale Liberalisierungspolitik besonders hart und unnachgiebig auf nationale Kultur- und Rechtstraditionen stößt, ist die Treuhänderschaft von Berufsgruppen und Professionen im Allgemeininteresse der Gesellschaft. Aus der Sicht des ökonomischen Wettbewerbsparadigmas handelt es sich dabei schlicht um Monopole, die es im Interesse der bestmöglichen Versorgung der Gesellschaft mit Dienstleistungen zu beseitigen und durch Wettbewerb zu ersetzen gilt.

Alle Urteile über die Zwangsläufigkeit des sich vollziehenden Wandels, die Befreiung von Monopolen, den Nutzen der daraus gezogen wird und die Fortschritte, die dabei erzielt werden, gehen von der Prämisse aus, dass der ökonomische Blick auf die Realitäten der einzig mögliche ist. Und es muss darauf vertraut werden, dass die Beseitigung der Berufsmonopole nicht zu anderen, aus der Ausübung von Marktmacht resultierenden Monopolen führt. Die Richtigkeit beider Annahmen ist nicht unbestritten. Nur aus einer rein ökonomischen Perspektive ist die Bestimmung von Bildungstiteln und die Definition von legitimer beruflicher Praxis in der Hand von Fachgesellschaften und Berufsverbänden ein Monopol, das den „Kunden“ eine Dienstleistung aufzwingt, das sie durch ihre Nachfrage nicht beeinflussen können. Dasselbe gilt aber auch für das Staatsmo-

26 Olson, M.: The Rise and Decline of Nations. Economic Growth, Stagflation, and Social Rigidities, New Haven, CT, 1982; Höpner, M.: Ist Politik gegen Verbände möglich? 25 Jahre Mancur Olsons „The Rise and Decline of Nations", in: Leviathan, 35/3 (2007), 310-347. 
nopol der Gesetzgebung, weshalb liberales Denken dem Staat auch so wenig Gesetzgebungskompetenz wie möglich überantworten will. Aus einer republikanischen Perspektive überlässt eine solche liberale Ordnung die fast nur noch als Wirtschaftsbürger (bourgeois) und kaum noch als Staatsbürger (citoyen) zu verstehenden Menschen der zu egoistischen Zwecken der individuellen Nutzenmaximierung ausgeübten Marktmacht der Starken. Diese naturwüchsige Vermachtung der Gesellschaft lässt sich im liberalen Sinn nur durch die Gewährleistung von Chancengleichheit und durch Gegenmacht zu jeder vorhandenen Macht vermeiden, im republikanischen Sinn nur durch ausreichende Gesetzgebungskompetenzen des Staates unter breiter Ausübung der demokratischen Rechte der Staatsbürger. In dieser Perspektive sind Märkte und Wettbewerb zur Herstellung und Allokation von Individualgütern geeignet, aber nicht zur Schaffung von Kollektivgütern.

Was als Individualgut und was als Kollektivgut zu gelten hat, versteht sich allerdings nicht von selbst. Es ist selbst Gegenstand politischer Auseinandersetzungen und letztlich der Entscheidung durch Gesetz, das heißt durch ein Kollektivgut höherer Ordnung, unterworfen. Liberale sehen kein Problem darin, Bildung als Individualgut komplett durch den Markt zu organisieren, für Republikaner ist das eine der wichtigsten öffentlichen Aufgaben, das heißt ein Kollektivgut, das ganz in der Hand des Staates und der demokratischen Gesetzgebung bleiben muss. Wissensmonopole von Fachgesellschaften und Berufsverbänden sind als eine dritte Form der Herstellung und Allokation von Gütern jenseits von Hierarchie (Staat) und Markt zu verstehen. Man kann sie als Vertrauensgüter bezeichnen. ${ }^{27}$ Dabei gilt es allerdings zu beachten, dass auch der Begriff „Vertrauensgut" zur Sprache der Ökonomie gehört und deshalb nur unzureichend erfasst, was unter der Entwicklung und Anwendung von Wissen in der Hand einer Fachgesellschaft oder eines Berufsverbandes zu verstehen ist. Bleiben wir aber zunächst bei diesem Begriff. Bei einem Vertrauensgut liegt die Fähigkeit zur Beurteilung der Qualität des Gutes ganz auf der Seite des „Produzenten“ bzw. „Vertrauensnehmers“. Der „Konsument“ bzw. „Vertrauensgeber“ muss sich vollständig auf den Produzenten verlassen. Im Extremfall kann der Vertrauensgeber überhaupt nicht zwischen verschiedenen Angeboten wählen, weil ihm dazu die Urteilsfähigkeit fehlt, ja sogar das Wissen darüber, ob er/sie das Produkt überhaupt benötigt. So zwingt der Staat beispielsweise Kinder mit Hilfe der

27 Barber, B.: The Logics and Limits of Trust, New Brunswick, NJ, 1983; Gambetta, D.: Can we Trust Trust?, in: ders. (Hg.): Trust: Making and Breaking Cooperative Relations, Oxford, 2000, 213-237. 
allgemeinen Schulpflicht zum Schulbesuch. Es geht hier um Treuhänderschaft. ${ }^{28}$ Der Staat nimmt die Rolle eines Treuhänders für den Lebensweg der Menschen wahr und überträgt die inhaltliche Ausübung der Treuhänderschaft auf die von ihm beauftragten Schulen und Lehrer. Eine besondere Bedeutung in der inhaltlichen Gestaltung dieser Treuhänderschaft kommt den Lehrerverbänden zu, die im Auftrag des Staates die Lehrpläne bestimmen. Für das Universitätsstudium haben bisher die Fachgesellschaften - etwa der Maschinenbauingenieure, der Chemiker oder der Soziologen - diese Treuhänderschaft übernommen. ${ }^{29}$

Die Treuhänderschaft für Studiengänge wird gegenüber der gesamten Öffentlichkeit ausgeübt, die in konkreten Fällen von den Wissenschaftsministerien repräsentiert wird. Soweit das Vertrauen in die Treuhänder da ist, lassen sich die Ministerien von den Fachgesellschaften sagen, was in eine Diplomprüfungsordnung hineingehört und was ein Diplomingenieur wissen muss, um selbst wiederum in der beruflichen Praxis die treuhänderische Rolle gegenüber seinen Auftraggebern bzw. Vertrauensgebern spielen zu können. Das beinhaltet immer, dass der Treuhänder weiß, was sein Auftraggeber zu wollen hat. Dementsprechend entscheiden nicht die Schüler oder Studenten darüber, was sie zu lernen haben, sondern die Lehrer und die Professoren in der delegierten Treuhänderschaft ihrer Fachgesellschaft. Sie sind in erster Linie gegenüber ihrer Fachgesellschaft verantwortlich, gegenüber ihren Schülern oder Studierenden nach Maßgabe der von ihrer Fachgesellschaft bestimmten Anforderungen. Ein gewisses Maß von dieser Treuhänderschaft steckt auch noch im Verhältnis zwischen den Fachgesellschaften und den privatwirtschaftlichen Unternehmen, die Absolventen mit Diplom einstellen. Was ihre Beschäftigten wissen müssen, wird vorrangig von den Fachgesellschaften und nicht von den Arbeitgebern bestimmt. Letztere können natürlich darauf drängen, an der Ausarbeitung von Lehrplänen beteiligt zu werden. Auf dem Niveau der dualen betrieblich/schulischen Ausbildung von Facharbeitern versteht sich das von selbst, aber nicht so sehr auf dem Niveau universitärer Studiengänge. Auf diesem Niveau haben die Fachgesellschaften das Heft in der Hand.

Das Besondere an dieser Art der Treuhänderschaft ist ihre Ausrichtung auf das Gemeinwohl, dem auch das Interesse der Klienten der professionellen Experten

28 Flannigan, R.: The Fiduciary Obligation, in: Oxford Journal of Legal Studies, 9/3 (1989), 285-294; Rodwin, M.A.: Strains in the Fiduciary Metapher: Divided Physician Loyalties and Obligations in a Changing Health Care System, in: American Journal of Law and Medicine, 21/2-3 (1995), 241-258.

29 Parsons, T./Platt, G. M.: Die amerikanische Universität, Frankfurt/M., 1990, 176-179, 523. 
untergeordnet wird. ${ }^{30}$ Das Interesse des Studierenden könnte sein, mit geringstem Aufwand das bestmögliche Zertifikat zu erhalten. Im Interesse des Gemeinwohls ist das jedoch nicht. Ein auf diese Weise schlecht ausgebildeter Arzt oder Ingenieur könnte erheblichen Schaden anrichten. Deswegen ist es im Interesse des Gemeinwohls, dass die Definition über das zum Erwerb eines Bildungstitels notwendige Wissen in der Hand der professionellen Experten bleibt. Die Kontrolle darüber, ob das im Einzelfall auch tatsächlich geschieht, kann wiederum nur bei denjenigen liegen, die das auch beurteilen können, also bei den professionellen Experten selbst. Dass daraus keine Willkür einzelner Experten entsteht, wird durch ihre Organisation in Fachgesellschaften gewährleistet, die kollektiv verbindliche Standards guter Praxis festlegen. Das ist Kontrolle durch peer review.

Gibt es einen Verdacht der Abweichung von den Standards, dann wird durch peer review geklärt, ob der Verdacht begründet ist. Das Handwerk und die klassischen Professionen der Ärzte und Anwälte sind in Kammern organisiert, die genau diese öffentliche Aufgabe der Standardsetzung und Selbstkontrolle wahrnehmen. Diese Selbstorganisation und Selbstkontrolle im öffentlichen Auftrag hat in Deutschland in besonders ausgeprägter Form handwerkliche und professionelle Dienstleistungen dem Denken in ökonomischen Kategorien von Tausch, Wettbewerb und Markt entzogen. Die Ausübung der entsprechenden Tätigkeiten ist deshalb strengen Zugangsberechtigungen unterworfen worden. Bei zahlreichen Handwerksberufen (insgesamt 41) können in Deutschland auch nach der Novellierung des Handwerksrechts im Jahre 2004 nur Meister selbständig einen Betrieb führen und Lehrlinge ausbilden. Kritiker halten diese Regelung für unvereinbar mit dem europäischen Recht. Die Ärzte benötigen die Approbation durch den Staat, die Anwälte die Zulassung durch die Anwaltskammer. Als Begründung für diese strengen Zugangsbedingungen galten immer die hohe Gemeinwohlverpflichtung und das entsprechend erforderliche Wissen sowie das besondere Ethos des Berufes. Die Handwerkerehre, der hippokratische Eid der Ärzte und das Anwaltsethos bringen diese ethische Fundierung der professionellen Tätigkeit und die damit verbundene Treuhänderschaft für das Gemeinwohl zum Ausdruck. Das immer gegebene Erwerbsinteresse wird durch diese treuhänderische Deutung und Organisation der handwerklichen und professionellen

30 Parsons, T.: The Professions and Social Structure, in: Social Forces, 17/4 (1939), 457-467; ders.: Professions, in: Sills, D. L. (Hg.): International Encyclopedia of the Social Sciences, Bd. 12, New York, $1968,536-547$. 
Tätigkeit in engen Grenzen gehalten. Es wird bei den Ärzten und Anwälten deshalb nicht nach Preisen, sondern nach einer Gebührenordnung abgerechnet. ${ }^{31}$

Dieses Modell der Treuhänderschaft ist allerdings in den vergangenen 30 Jahren zunehmend unter Beschuss geraten. Dabei haben sich zwei Entwicklungen gegenseitig unterstützt. Die Vergrößerung der Zahl professioneller Experten hat deren Tätigkeit in gewissem Maße profanisiert und dadurch aus der Treuhänderschaft heraus und in die Erwerbstätigkeit hineingezogen. Die Zahl der Verletzungen von Standards ist mit der Zahl der Experten und der Zahl von Dienstleistungen zwangsläufig gewachsen. Infolgedessen mussten häufiger die Gerichte des Staats beispielsweise den Rechten von Patienten zur Geltung verhelfen, nachdem dies von den Kammergerichten der Ärzte nicht mehr lückenlos gewährleistet wurde. Parallel dazu hat mit der weltweiten Wende hin zu neoliberalem Ordnungsdenken seit den 1980er Jahren und der massiven Expansion von Unternehmensberatungen sowie ihrem Eindringen in alle Funktionsbereiche der Gesellschaft ökonomisches Denken in Kategorien von Effizienz, Wettbewerb und Märkten an Bedeutung gewonnen.

Im Zuge des Ineinandergreifens von Profanisierung und Ökonomisierung wurde auch die professionelle Dienstleistung mehr als zuvor als eine Erwerbstätigkeit betrachtet, die ein Individualgut bereitstellt, für dessen Qualität der Wettbewerb auf Märkten am besten sorgen kann. Das gilt auch für Forschung und Lehre an den Universitäten. ${ }^{32}$ Für diesen Zweck benötigt man aufgeklärte und informierte Kunden. Das sollen Evaluationen und Rankings leisten. Und die Kunden müssen Rechte haben, um sich gegen schlechte Dienste wehren zu können. Diesen Zweck erfüllen Kundenbewertungen, von dem schnell ausgefüllten Zettel zum Zimmerservice im Hotel über die Bewertung der Betreuung durch den Meister beim Automobilkundendienst bis hin zur Lehrevaluation an den Hochschulen und zur öffentlich gemachten Bewertung ärztlicher Leistungen durch die Patienten. Für dieses Denken in ökonomischen Kategorien ist es deshalb nahe liegend, dass über die Gestaltung von Studiengängen nicht mehr ausschließlich die Fachgesellschaften entscheiden, sondern die Studierenden als primäre und die Arbeit-

31 Abbot, A.: The System of Professions. An Essay on the Division of Expert Labor, Chicago, 1988.

32 Rhoades, G.: Managed Professionals. Unionized Faculty and Restructuring Academic Labor, Albany, 1998; Finkelstein, M.J./Seal, R.K./Schuster, J.H.: The New Academic Generation. A Profession in Transformation, Baltimore, MD, 1998. 
geber als sekundäre Kunden. Am besten lässt man in dieser Perspektive einen Bildungsmarkt über die Allokation von Angebot und Nachfrage entscheiden. ${ }^{33}$

Für den ökonomischen Blick auf die Welt sind Treuhänderschaften nichts anderes als Monopole, die es im Interesse einer passgenauen Allokation von Angebot und Nachfrage aufzulösen gilt. Das ist im rein ökonomischen Sinn richtig, in einer weiteren Perspektive jedoch einseitig, weil wesentliche Elemente der Treuhänderschaft nicht beachtet werden. Dazu gehört insbesondere die Tatsache, dass professionelle Dienstleistungen nicht per se handelbare Individualgüter sind, sondern nur dann, wenn ihre Gemeinwohlverpflichtung ausgeblendet wird. Außerdem muss ignoriert werden, dass der Handel von Individualgütern auf einem Markt aus sich heraus kein Gemeinwohl hervorbringen kann. Weiterhin muss man übersehen, dass Treuhänderschaft eine Form der Institutionalisierung der ethischen Bindung an das Gemeinwohl darstellt, zu der das Monopol über die Dienstleistung zwangsläufig genauso gehören muss, um überhaupt wirksam werden zu können, wie das Gewaltmonopol zum Staat zwecks Ausübung seiner Gesetzgebungskompetenz.

Bevor wir auf dieser Linie die ökonomische Kritik am Monopol der Treuhänderschaft als unangemessen zurückweisen, muss allerdings konstatiert werden, dass auch das treuhänderische Monopol wie alle Monopole Angebotsbeschränkungen beinhaltet, die weder dem öffentlichen Interesse noch dem privaten Interesse der Klienten dienen. ${ }^{34}$ Im Hochschulbereich ist es die Vielfalt von Studienangeboten, die unter dem Monopol der Fachgesellschaften leidet. Das gilt umso mehr, je weniger die Fachgesellschaften intern differenziert sind. Neues Wissen aus der Peripherie hat es schwer, ins Zentrum der Prüfungsordnungen vorzustoßen. Man denke nur an die lange Zeit erfolgreiche Abwehr psychosomatischer Erkenntnisse durch die Schulmedizin. Die Fachgesellschaften sind die besonders strengen Hüter der Dogmen einer Disziplin. Im Vergleich zu den USA sticht die deutsche Universitätsausbildung durch eine besonders strenge und am dogmatischen Kern von Disziplinen hängende Ausrichtung der Studiengänge hervor. Das hat insbesondere die Entwicklung interdisziplinärer Studien behindert und ist zu einem handfesten Nachteil in der Ausdifferenzierung von feingliedriger Vernetzung von Disziplinen geworden. Die Kehrseite der stolzen Tradition von Diplomen ist

33 Lohmann, I.: Commercialism in Education. Historical Perspectives, Global Dimensions and European Educational Research Fields of Interest, in: European Educational Research Journal, 1/3 (2002), 550565.

34 Freidson, E.: Profession of Medicine. A Study of the Sociology of Applied Knowledge, Chicago, 1970/1988. 
die Konservierung des disziplinären Wissens und die Behinderung von inter- und transdisziplinärem Denken. Der deutsche Diplomingenieur ist besonders gut in der Perfektionierung seines Wissens, aber schlecht vorbereitet auf die Erweiterung seines Wissens in den Grenzbereichen zwischen den Disziplinen. Neuschöpfungen wie der Diplom-Wirtschaftsingenieur sind lange Zeit solitäre Studiengänge ohne größere Ausbreitung geblieben. Das sind die richtigen Voraussetzungen für inkrementale Innovationen in herkömmlichen Technologien, aber die falschen für radikale Innovationen in Spitzentechnologien. ${ }^{35}$

Der Tendenz zum Dogmatismus entgegenzuwirken, verlangt eine offene, pluralistische und wettbewerbsintensive Gestaltung der Fachgesellschaften mit Untergliederungen nach einer Vielzahl von Fachgebieten mit Überschneidungen in den Grenzbereichen zwischen den Disziplinen. Die Kooperation der Fachdisziplinen in gemeinsamen Forschungsprojekten und Studiengängen unterstützt diese Politik gegen die dogmatische Erstarrung des Wissens in den Zwangsjacken der Disziplinen und ihrer Fachgesellschaften. Gleichwohl geht mit der Pluralisierung der Studiengänge zwangsläufig eine Entberuflichung der Erwerbstätigkeiten und eine Vermarktlichung der Allokation von Bildung und Beschäftigung einher. An die Stelle des verberuflichten Arbeitnehmers der fordistischen Ära mit seinem Beschäftigungssicherheit gewährleistenden Wissensmonopol tritt der Arbeitskraftunternehmer des Postfordismus, dessen asset weniger ein Monopol über Wissen und mehr die Kompetenz zur Selbstvermarktung ist. Der Arbeitskraftunternehmer zeichnet sich durch Selbstkontrolle über seine Tätigkeit, Ökonomisierung seines Verhaltens und Verbetrieblichung seiner Lebensführung aus. ${ }^{36}$ Auf diesen neuen Typus treffen alle von Richard Sennett beschriebenen Unstetigkeiten des Driftens in der Lebensführung ${ }^{37}$ zu. Mit der Unsicherheit in der Lebensplanung wächst auch die Fragilität der Gesellschaft. ${ }^{38}$

Bei allen Reformmaßnahmen ist zu beachten, dass sich die davon betroffenen Akteure strategisch darauf einstellen, um den größtmöglichen Nutzen für sich zu erlangen. Das gilt gerade dann, wenn Akteure in ökonomischen Kategorien beg-

35 Hall, P./Soskice, D.: Varieties of Capitalism, Oxford, 2001; Münch, R.: Das Regime des liberalen Kapitalismus. Inklusion und Exklusion im neuen Wohlfahrtsstaat, Frankfurt/New York, 2009.

36 Voß, G. G./Pongratz, H.J.: Der Arbeitskraftunternehmer. Eine neue Grundform der Arbeitskraft?, in: Kölner Zeitschrift für Soziologie und Sozialpsychologie, 50/1 (1998), 131-158; Pongratz, H.J./Voß, G. G.: Arbeitskraftunternehmer. Erwerbsorientierungen in entgrenzten Arbeitsformen, Berlin, 2003; Pongratz, H.J.: Eine Gesellschaft von Unternehmern. Expansion und Profanierung schöpferischer Zerstörung in kapitalistischen Ökonomien, in: Berliner Journal für Soziologie, 18/3 (2008), 457-475.

37 Sennett, R.: Der flexible Mensch. Die Kultur des neuen Kapitalismus, Berlin, 1998.

38 Münch, R.: Das Regime des liberalen Kapitalismus, a. a.O. 
riffen werden und per self-fulfilling prophecy auch ökonomisch rational handeln, um ihren Eigennutz zu maximieren. Der ethisch motivierte homo professionalis verwandelt sich auf diesem Weg in einen homo oeconomicus. „Gaming the system " rational kalkulierender Akteure kann Reformmaßnahmen leicht zu einer ganz anderen Wirklichkeit werden lassen als ursprünglich gedacht. ${ }^{39}$ Allerdings ist auch die traditionelle professionelle Tätigkeit davon nicht frei, und zwar umso mehr, je mehr die professionelle Lehre angesichts von Alternativen zur Orthodoxie gerät und mittels Monopolstellung verteidigt wird. Bei der Umstellung professioneller Tätigkeiten auf den Marktmechanismus besteht wiederum die Gefahr, dass Marktmacht Alternativen verdrängt und die Dominanz des Erwerbsinteresses jegliche Gemeinwohlverpflichtung zum Verschwinden bringt.

\section{Weshalb transnationale Regierung und Verwaltung die Vermarkt- lichung von Professionen favorisiert}

Bei der Vermarktlichung der Professionen treffen die identifizierten Kräfte der Transnationalisierung von Regierung und Verwaltung zusammen. Die Professionen sind Träger nationaler Traditionen der Treuhänderschaft. Im nationalen Feld der Politik konnten sie sich auf die gesetzliche Garantie ihrer Selbstorganisation stützen, die ihnen quasi eine öffentlich-rechtliche Autorität verliehen hat. In gewissem Maße hat sie diese staatliche Weihe unantastbar gemacht. Im politischen Feld waren konservative Parteien die Stützen der Professionen, und zwar aufgrund ihres Denkens, dass Berufsstände die Marktkräfte bändigen und der Gesellschaft eine berechenbare Ordnung geben. Die Professionen hatten dadurch Zugang zu gewichtigem politischem Kapital, das heißt zu Entscheidungsmacht. Mit der Transnationalisierung der Regulierung von Dienstleistungen wird der professionellen Treuhänderschaft ihre traditionale Legitimität entzogen. Die Debatten über das General Agreement on Trade in Services (GATS) und die Trade Related Aspects of Intellectual Property Rights (TRIPS) der Welthandelsorganisation (WTO) sowie über die europäische Dienstleistungsrichtlinie haben diesen Konflikt zwischen transnationalen Rationalitäten und lokalen Traditionen klar zutage gefördert. ${ }^{40}$ Außerdem verliert das ihnen im nationalen Kontext zu-

39 Langer, R.: Nicht Wissen hilft - Evaluation in der Konkurrenz von Symbolisierungen, Wiesbaden, 2008.

40 Lauer, R./Fasel, S.: Negotiating Trade in Educational Services within the WTO/GATS Context, in: Außenwirtschaft, 59/II (2003), 279-208; Davies, G.: The Services Directive: Extending the Country of Origin Principle and Reforming Public Administration, in: European Law Review, 32/2 (2007), 232- 
gängliche politische Kapital im offenen transnationalen Kontext an Wert. In diesem Kontext gibt es keine traditionale Legitimität, weil alles historisch Gewachsene nationalen Ursprungs ist. Hier muss alles neu verhandelt werden, und das kann allein unter dem Gesichtspunkt der Funktionalität einer Ordnung (einer Regelung, einer Richtlinie) und im Spannungsfeld unterschiedlicher, von den Regierungen vertretener Interessen geschehen. So können die Interessen an der Erhaltung professioneller Treuhänderschaft von konservativen Regierungen in transnationale Verhandlungen eingebracht werden, dort sind sie aber ihrer traditionalen Legitimität beraubt und müssen sich als blanke Interessen behaupten. Außerdem sind transnationale Verhandlungen in aller Regel auf eine spezifische Funktion, vorrangig auf die Erleichterung des transnationalen Wirtschaftsverkehrs, bezogen. Das gilt für die Europäische Union (EU) und erst recht für die WTO. Dagegen sind umweltpolitische Regulierungen viel weniger auf Dauer gestellt und eine Sache zweckspezifischer Abkommen, wie etwa das OzonRegime und das Klimaschutzregime. Sozialpolitische Abkommen - wie die Konvention der International Labor Organization (ILO) - sind nicht sanktionsbewehrt und deshalb noch weniger bedeutsam.

Für unsere Fragestellung ist besonderes relevant, dass für die EU und erst recht die WTO die aufkommenden Fragen in erster Linie unter dem funktionalen Gesichtspunkt der Erleichterung des Wirtschaftsverkehrs zu behandeln sind. Nach Art. 30 EGV haben die Mitgliedstaaten der EU nur unter sehr eingeschränkten Bedingungen das Recht zu Regulierungen, die den freien Wirtschaftsverkehr behindern. Es müsste nachweislich die Gesundheit der Bevölkerung oder die öffentliche Ordnung gefährdet sein. Ob dieser Nachweis gelingt, entscheidet der Europäische Gerichtshof, der sich nur von sachlich stichhaltigen, letztlich wissenschaftlich begründeten und deshalb allgemein, unabhängig von Kultur- und Rechtstraditionen gültigen Nachweisen überzeugen lässt. ${ }^{41}$ Demgemäß war es eher eine Überraschung, dass der Gerichtshof im Mai 2009 den Schutz der deutschen Apotheken vor kommerzieller Konkurrenz unangetastet ließ. Wegen der funktionalen Spezifikation ist die transnationale Rechtssetzung aus der auf nationalstaatlicher Ebene gegebenen Verflechtung mit Traditionen, Privilegien und Interessen aller Art und der damit verbundenen Notwendigkeit der politischen Koordination herausgelöst und kann deshalb nachhaltiger von Experten nach

245; Nikolaïdis, K./Schmidt, S. K.: Mutual Recognition on "Trial”: The Long Road to Services Liberalization, in: Journal of European Public Policy, 14/5 (2007), 717-734.

41 Craig, P./de Búrca, G. (Hg.): EU Law: Text, Cases and Materials, Oxford, 2003, 659-673; Stone Sweet, A.: The Judicial Construction of Europe, Oxford, 2004, 109-145. 
funktionsspezifischer Zwecksetzung bestimmt werden, solange keine massiven Interessen im Spiel sind, die von gewichtigen nationalen Regierungen vertreten werden. Dabei kann es sich aber auf lange Sicht nur um Interessen handeln, die gegenüber dem primären Interesse an Marktöffnung einen sekundären Status einnehmen und deshalb nur als Begleitprogramm zur Marktschaffung mitlaufen. Das gilt für Regulierungen zur Sicherheit von Lebens- und Arzneimitteln genauso wie für die Gesundheitsrisiken am Arbeitsplatz oder für den Umweltschutz. Auch die Sozialpolitik und das Arbeitsrecht nehmen einen gegenüber der Marktschaffung sekundären Rang ein, wie etwa der Europäische Gerichtshof erst jüngst in einigen spektakulären Urteilen zur Wahrnehmung von Arbeitnehmerrechten mittels Streik ${ }^{42}$ und zum Tarifrecht ${ }^{43}$ deutlich gemacht hat. ${ }^{44}$

Die funktionale Ausrichtung der transnationalen Politik auf Marktschaffung verlangt vor allem ökonomischen Sachverstand. Unter der Leitlinie der Marktschaffung ist es naheliegend, dass ökonomischer Sachverstand in erster Linie die Beseitigung von Marktschranken und von Hindernissen des Wettbewerbs verlangt. Und weil die Marktschaffung das erklärte Ziel der EU ist, liegt es nahe, dass auch der juristische Sachverstand des EuGH das Schleifen von Monopolen betreibt. Transnationale Integration favorisiert demnach eine Politik der Marktschaffung, die in die Nationalstaaten hineinwirkt und Kultur- wie Rechtstraditionen die Legitimität entzieht, die sich nicht dem Markt- und Wettbewerbsparadigma fügen, sondern einer anderen Logik folgen.

Mit dieser funktionalen Favorisierung des Markt- und Wettbewerbsparadigmas im transnationalen Kontext geht ein Gewinn an Definitionsmacht derjenigen politischen Kräfte einher, die von sich aus für eine liberale Ordnung stehen, und zwar auf der transnationalen und der nationalen Ebene. Liberale Regierungen und Parteien gewinnen an Gewicht. So erhält beispielsweise in Deutschland die von liberalen Kräften schon länger favorisierte Liberalisierung der Berufsbildung durch die EU Auftrieb und kann auf der nationalen Ebene Erfolge erzielen, die ohne die Transnationalisierung des Politikfeldes der Berufsbildung und die ent-

42 EuGH, Urt. v. 11.12. 2007, Rs. 438/05 (Viking), Slg. 2007, I-10779; EuGH, Urt. v. 18. 12. 2007, Rs. 341/05 (Laval), Slg. 2007, I-11767.

43 EuGH, Urt. v. 03.04. 2008, Rs. 346/06 (Rüffert), Slg. 2008, I-1989; EuGH, Urt. v. 19.06. 2008, Rs. 319/06 (Luxemburg), Slg. 2008, I-4323.

44 Höpner, M.: Usurpation statt Delegation. Wie der EuGH die Binnenmarktintegration radikalisiert und warum er politischer Kontrolle bedarf, MPIfG Discussion Paper 08/12, 2008; Scharpf, F. W.: Legitimität im Europäischen Mehrebenensystem, in: Leviathan 37/2 (2009), 244-280. 
sprechende Verschiebung von symbolischer Macht sowie den damit einhergehenden Wandel der Legitimationsstrukturen nicht möglich gewesen wären. ${ }^{45}$

\section{Wie kann dem Konflikt zwischen transnationalen Rationalitäten und nationalen Traditionen institutionell Rechnung getragen werden?}

Eine entscheidende Frage ist an dieser Stelle, ob die Nationalstaaten tatsächlich die Möglichkeit hätten, dem Primat der Marktschaffung entgegenzutreten und sich seinem Diktat zu entziehen. Folgen wir Durkheims klassischer Studie zur Arbeitsteilung ${ }^{46}$, dann ist das nur auf Kosten verschärfter Verteilungskonflikte zwischen den und innerhalb der nationalen Gesellschaften möglich. Am Ende des Zweiten Weltkrieges war das endgültig klar geworden, weshalb damals in der Tat die Weichen für die zunehmende internationale Arbeitsteilung gestellt wurden, zuerst in Europa, dann auch in anderen Regionen der Welt und im globalen Rahmen durch die WTO. Das Ende des Kalten Krieges hat dieser Entwicklung einen kräftigen Schub gegeben. Der Spielraum für die Aufrechterhaltung nationaler Kultur- und Rechtstraditionen ist deshalb nicht sehr groß. Die Traditionen haben nur eine Überlebenschance, wenn sie mit der Marktschaffung nicht im Konflikt stehen oder wenn ihre Notwendigkeit zum Schutz der Gesundheit der Bevölkerung und der öffentlichen Ordnung sachlich hieb- und stichfest, allgemein gültig, das heißt kulturunabhängig nachgewiesen werden kann. Das gilt auf jeden Fall für die EU. Für die WTO-Mitglieder ist der Spielraum größer, weil die WTO nicht den supranationalen Status wie die EU hat, sondern noch mehr eine Vereinigung souveräner Staaten ist. Den Mitgliedstaaten der WTO ist es beispielsweise erlaubt, Industrien zu schützen, wenn ihr Zusammenbruch zu erheblichen Verwerfungen wie etwa nicht mehr auszugleichenden Massenentlassungen führen würde. ${ }^{47}$

Allerdings hat die Beseitigung nationaler, regionaler und lokaler Traditionen keineswegs immer zur Folge, dass die neuen Regeln so wirken, wie es intendiert war, allein schon aufgrund der ganz unterschiedlichen Kontextbedingungen und des unvermeidlichen gaming the system. Insbesondere wirkt die schablonenhafte

45 Schmidt, S.K.: Die nationale Bedingtheit der folgen der Europäischen Integration, in: Zeitschrift für internationale Beziehungen, 10/1 (2003), 43-68; Trampusch, C.: Jenseits von Anpassungsdruck und Lernen: die Europäisierung der deutschen Berufsbildung, in: ZSE, 6/4 (2008), 577-605.

46 Durkheim, É., a. a. O.

47 Langer, S.: Grundlagen einer internationalen Wirtschaftsverfassung, München, 1995. 
Anwendung ein und derselben Regelungen und Instrumente auf eine Auflösung existierender Praktiken hinaus, ohne dass die neuen Regeln notwendigerweise bessere Ergebnisse erbringen. Es besteht immer die Gefahr, dass sich Alt und Neu so zu einem Hybrid verbinden, dass die alten Traditionen ausgehöhlt werden und die neuen Regelungen kontraproduktiv wirkende Nebeneffekte erzeugen, die man nicht vorausgesehen hat. Gegenwärtig sind solche Erscheinungen im Feld der Bildungspolitik im Gefolge des PISA-benchmarkings der OECD und des europäischen Bologna-Prozesses zu beobachten. ${ }^{48}$

Will man solche Funktionsdefizite und die damit einhergehenden Legitimationskonflikte in bewältigbaren Grenzen halten, dann muss konsequent geklärt werden, was überhaupt der inter- bzw. supranationalen Regulierung bedarf. Dazu sind insbesondere die neuen Formen der Regierung und Verwaltung durch benchmarking, monitoring und best practices zu zählen, zumal von ihnen nachhaltige Anpassungszwänge ausgehen und nationale Kultur- und Rechtstraditionen sowie mit ihnen ihre besonderen Leistungen außer Kraft gesetzt werden, ohne dass es dafür einen unbestreitbaren Nachweis der Notwendigkeit gibt. Das gilt etwa für PISA und Bologna. Die Reflexion über den Wert und Nutzen nationaler, regionaler und lokaler Traditionen würde auch dadurch befördert, dass die Koordination zwischen den nationalen Regierungen größeren Spielraum erhält und den nationalen Parlamenten eine angemessene Mitsprache bei der transnationalen (europäischen) Rechtssetzung gewährt wird. ${ }^{49}$

Angesichts der Überlagerung legitimer nationaler Strukturen der Herrschaft durch nicht-legitime transnationale Strukturen herrscht ziemliche Ratlosigkeit darüber, wie es weiter gehen soll. Ein Ausweg aus dem Dilemma der funktionalen Notwendigkeit der Transnationalisierung von Entscheidungskompetenzen und dem damit einhergehenden Aufbau nicht-legitimer Formen der Herrschaft ist nicht in Sicht. Regieren im europäischen Mehrebenensystem lässt sich nicht nach

48 Saarinen, T.: "Quality" in the Bologna Process: From "Competitive Edge" to Quality Assurance Techniques, in: European Journal of Education, 40/2 (2005), 189-204; Keeling, R.: The Bologna Process and the Lisbon Research Agenda: the European Commission's Expanding Role in Higher Education Discourse, in: European Journal of Education, 41/2 (2006), 203-223; Ravinet, P.: From Voluntary Participation to Monitored Coordination: Why European Countries Feel Increasingly Bound by their Commitment to the Bologna Process, in: European Journal of Education 43/3 (2008), 353-367; Münch, R.: Globale Eliten, lokale Autoritäten. Bildung und Wissenschaft unter dem Regime von PISA, McKinsey \& Co, Frankfurt/M., 2009.

49 Hesse, J.J./Grotz, F.: Europa professionalisieren. Kompetenzordnung und institutionelle Reform im Rahmen der Europäischen Union, Berlin, 2005. 
dem Muster einer Mehrheitsdemokratie kontrollieren. Es bedarf einer Kontrolle nach dem Muster von checks and balances.

Ein wesentlicher Bestandteil der Kontrollen und Gegenkontrollen beinhaltet die Abstimmung transnationaler (europäischer) Regulierungen mit nationalen Kultur- und Rechtstraditionen, und zwar schon deshalb, weil das in diesen Traditionen steckende Erfahrungswissen und Problemlösungspotential nicht vollständig durch globale Rationalitätsmodelle ersetzt werden kann und weil möglicherweise nationale, regionale oder lokale Praktiken in ihrem jeweiligen Umfeld bessere Leistungen erbringen als die Implementation globaler Einheitsmodelle. Mit der kulturellen Diversität ist es wie mit der Biodiversität. Sie wird durch globales bzw. europäisches benchmarking und monitoring und durch die Orientierung an best practices gefährdet, ihr Erhalt ist jedoch bedeutsam für die Offenheit der sozio-kulturellen Evolution. Wenn demgemäß die nationalen Regierungen weiterhin Kompetenzen behalten und europäische Entscheidungsprozesse nach wie vor von internationaler Koordination kontrolliert werden, und wenn nationale Parlamente gewichtiger mit europäischen Fragen befasst werden, dann tragen solche Maßnahmen zur Erhaltung von kultureller Diversität bei. Das schränkt gewiss die Chancen zu supranationaler Handlungsfähigkeit ein, bedeutet aber nicht zwangsläufig, dass es für die unabdingbar transnational (europäisch) zu lösenden Probleme keine Lösung jenseits der Koordination zwischen souveränen Staaten gibt.

Die funktional spezifische Ausdifferenzierung supranationaler Entscheidungskompetenzen kann mit der bewussten Belassung von Entscheidungskompetenzen auf nationaler, regionaler oder lokaler Ebene einhergehen, wo es um die Einfügung von Maßnahmen in die jeweiligen Kontexte und Traditionen geht. So ist es in der Tat eine der Klärung bedürftige Frage, ob alle Bildungssysteme einheitlich am PISA-Test oder an den Bologna-Kriterien gemessen werden müssen, nur deshalb weil die Nationen miteinander einen gemeinsamen Wirtschaftsraum teilen. Anders als bei der Abwicklung von Geschäften besteht bei der Bildung nicht notwendigerweise ein Angleichungszwang. Im Gegenteil, das gegenseitige Anregungspotential ist bei intensivem Kulturaustausch umso größer, je divergenter sich die Kulturen darstellen. Dass solche Einwände gegen das PISA-Verfahren und den Bologna-Prozess in die undankbare Rolle des Plädoyers für alte Zöpfe und Privilegien gedrängt werden, ist wohl durch die konstatierte Dominanz von einseitig ökonomischen Denkmodellen im transnationalen Kontext zu erklären. Das heißt aber nicht, dass sie nicht ernst zu nehmen sind. 